\title{
Evaluation of Three Rapid Diagnostic Test Kits in the Diagnosis of Plasmodium Species Infections among Primary School Children in Baringo County, Kenya
}

\author{
Collince Jared Omondi', Lucy Muthony Kamau' ${ }^{1}$, Humphrey Kariuki Njaanake ${ }^{2}$, \\ Douglas Anyona ${ }^{3}$, Daniel Onguru ${ }^{3 *}$, Benson Estambale ${ }^{3}$ \\ ${ }^{1}$ Department of Zoological Sciences, Kenyatta University, Nairobi, Kenya \\ ${ }^{2}$ Department of Medical Microbiology, College of Health Sciences, University of Nairobi, Nairobi, Kenya \\ ${ }^{3}$ School of Health Sciences, Jaramogi Oginga Odinga University of Science and Technology, Bondo, Kenya \\ Email: ^danonguru@yahoo.com
}

How to cite this paper: Omondi, C.J., Kamau, L.M., Njaanake, H.K., Anyona, D., Onguru, D. and Estambale, B. (2017) Evaluation of Three Rapid Diagnostic Test Kits in the Diagnosis of Plasmodium Species Infections among Primary School Children in Baringo County, Kenya. Open Journal of Clinical Diagnostics, 7, 31-44.

https://doi.org/10.4236/ojcd.2017.71004

Received: October 22, 2016

Accepted: March 25, 2017

Published: March 28, 2017

Copyright (c) 2017 by authors and Scientific Research Publishing Inc. This work is licensed under the Creative Commons Attribution International License (CC BY 4.0).

http://creativecommons.org/licenses/by/4.0/

\begin{abstract}
Background: Due to the difficulty in implementing microscopy-based definitive diagnosis of malaria especially in resource-poor areas, rapid diagnostic test (RDT) kits as alternatives have been developed by different companies. Kits from different companies may differ in sensitivity and specificity. It is therefore imperative to conduct field evaluation of kits from different manufacturers before they are recommended for widespread use in diagnosis of malaria. The present study sought to evaluate the performance of malaria RDT kits from two manufacturers against microscopy in detection of Plasmodium spp. infections among primary school children in Baringo County, Kenya. Methods: Two cross-sectional comparative surveys to assess three RDT kits (CareStart HRP-2, SD Bioline Ag-Pf and SD Bioline Ag-Pf/Pan) were conducted on 561 pupils from three primary schools within the Kerio Valley of Baringo County, Kenya. Results: A total of 561 blood samples were tested for Plasmodium spp. within a period of four months. SD Bioline Ag-Pf/ Pan RDT kit had a higher sensitivity (90\%) compared to that of CareStart HRP-2 (70\%) but this was not statistically significant (McNemar's $\chi^{2}=0.5$, df $=1, p=0.4795)$. The sensitivity of SD Bioline Ag-Pf was the same as that of Care Start HRP-2 (82.4\%). The number of positive samples by microscopy was relatively lower than those detected by RDT kits. Two cases of non- $P$. falciparum infections were detected by RDT kits during the second survey. Conclusion: The results of the present study suggest that the performance of the three kits in the diagnosis of $P$. falciparum infections was better than microscopy. The sensitivity of SD Bioline Ag-Pf/Pan was slightly greater than that of Care Start HRP-2, although not statistically different.
\end{abstract}




\section{Keywords}

Rapid Diagnostic Tests, Microscopy, Malaria, Sensitivity, Specificity, Baringo

\section{Introduction}

Malaria is a major public health problem accounting for $21 \%$ of out-patient consultation and $3 \%-5 \%$ of hospital admissions in Kenya (KMOH [1] [2]). Children under the age of five years and pregnant women are the most affected with an estimated 34,000 children dying due to malaria each year in Africa [3]. Most of the malaria-related deaths occur in rural areas of the continent due to misdiagnosis resulting in inappropriate or late treatment [4]. In efforts to curb the high malaria-related mortality and morbidity, the Government of Kenya considered malaria intervention measures as a priority investment to achieve its 2030 vision [1] [2].

Accurate and timely diagnosis accompanied with prompt treatment is an important aspect of malaria management and control [5]. Unfortunately, many treated confirmed cases in Africa are lower compared to other regions of the world [4]. This suggests that majority of treated cases are clinically diagnosed yet this type of diagnosis can be unreliable due to the non-specific nature of signs and symptoms of malaria [5]. It may also lead to indiscriminate use of antimalarial drugs and compromise the required quality care for patients suffering from other diseases displaying symptoms and signs similar to those of malaria [6] [7] [8]. In addition, when transmission rates are low, clinical signs like fevers may not automatically point to Plasmodium spp. infection. Hence parasite-based diagnosis is a vital strategy to avoid inappropriate use of antimalarial drugs [9].

The WHO recommends that malaria case management be based on parasitological test diagnosis in all cases with exception of young children in endemic areas [6] [10]. Microscopic analysis of malaria parasites and use of rapid diagnostic tests (RDTs) to detect malaria parasite's antigen in blood, are the two diagnostics most likely to have a greater impact on malaria control today [11]. However, in most remote settings where adequate expert performance of microscopy is needed, this gold standard may be a challenge due to lack of basic microscopy infrastructure [12]. For application of other malaria diagnostic tests such as rapid diagnostic tests to circumvent, the drawbacks are therefore very necessary. Various RDT kits have been in use but their sensitivity and specificity are subject to field evaluation under different endemicity and transmission intensity settings before being recommended for detection of Plasmodium species infections [13]. This is important since their performance may be affected by a number of factors such as, extreme temperatures and high humidity (WHO, 2003), prozone effect [14] and cross-reactivity with human auto-antibodies [15]. In addition, persistence of parasite antigen in blood even after the clearance of malaria parasites by antimalarial drugs often leads to false positive RDT results [11]. 
Many malaria RDT kits are commercially available but their performance varies depending on the type of malaria antigen targeted for detection in blood [11] [16] [17]. In the present study, CareStart HRP-2, SD Bioline Ag-Pf and SD Bioline Ag-Pf/Pan RDT kits were evaluated to compare their diagnostic accuracy.

\section{Materials and Methods}

\subsection{Study Site}

This study was conducted in three primary schools located within three sub-locations (Barwessa, Kapluk and Keturwo) in the riverine zone of Baringo County. The riverine zone borders the Kerio River, which is to the extreme west of the county. In this area, the soils are poorly drained with a slope of less than $6 \%$ making it prone to flooding. Baringo County is located in the former Rift Valley province and lies between $35.602 \mathrm{E}, 0.541 \mathrm{~N}$ and $36.277 \mathrm{E}, 0.723 \mathrm{~N}$ at altitudes ranging between 870 to 2499 metres above sea level. It is mostly arid or semiarid, covering an area of $11,075.3 \mathrm{~km}^{2}$ with a total population of 555,561 as per the 2009 Kenya population and housing census (KNBS, 2009). The region has two distinct weather patterns with the temperatures in the southern part ranging between $25^{\circ} \mathrm{C}$ during the cold months and $30^{\circ} \mathrm{C}$ during the hot months. In the northern parts, temperatures range between $30^{\circ} \mathrm{C}$ and $35^{\circ} \mathrm{C}$. The county experiences two rainy seasons; the long rains (March to June) and the short rains (October to November). It receives between 1000 and $1500 \mathrm{~mm}$ of rainfall annually in the highlands and about $600 \mathrm{~mm}$ in the lowlands.

The most prevalent diseases in this region are malaria and upper respiratory tract infections. Malaria transmission in the region is seasonal and peaks during the rainy season. The current incidence of malaria is about $12 \%$ based on outpatient visits to local health facilities (KMOH, 2015).

\subsection{Study Design}

This was a cross-sectional comparative survey that sought to assess the performance of three RDT kits (CareStart HRP-2, SD Bioline Ag-Pf and SD Bioline Ag-Pf/Pan). Primary school pupils aged 5 to 15 years were examined for infection with Plasmodium spp. using the three kits, with smear microscopy as confirmatory test.

\subsection{Sample Size Determination}

To determine the performance of RDT kits, sample size was calculated using the formula given by Naing, Winn [18]. Briefly, using the malaria prevalence data of $12 \%$, precision 0.05 and significance level, the minimum number of pupils needed for this study was 162 . The sample size was calculated using the formula:

$$
n=\frac{z^{2} P(1-P)}{d^{2}}
$$

where; 
$n$-Sample size;

$z$-Confidence level of $95 \%$ (standard value of 1.96 );

$P$-Estimated malaria prevalence (12\%);

$d$-Margin of error of $5 \%$ (standard value of 0.05 ).

$$
\begin{aligned}
n & =\frac{1.96^{2} * 0.12 * 0.88}{0.05^{2}} \\
& =162.2692=162
\end{aligned}
$$

The total number of pupils screened in the first and second surveys was 261 and 300 respectively.

\subsection{Sampling Technique}

Three primary schools within the riverine zone of the study area were selected based on their proximity to malaria vector breeding habitats. A total of $300 \mathrm{pu}$ pils aged between 5 and 15 years were recruited after obtaining informed consent from their parents or guardians.

\subsection{Collection and Examination of Blood Samples}

A finger prick blood sample $(20 \mu \mathrm{l})$ was collected from each pupil after a clinical examination and recording of the signs or symptoms. About $5 \mu \mathrm{l}$ of the blood sample was examined for presence of malaria using SD Bioline Ag-Pf or SD Bioline Ag-Pf/Pan kit and another $5 \mu \mathrm{l}$ for CareStart HRP-2 kit. The other $10 \mu \mathrm{l}$ was used to prepare both thin and thick smear for examination under a light microscope.

\subsection{Rapid Diagnostic Test}

SD Bioline Ag-Pf/Pan (Cat no. 05FK60), SD Bioline Ag-Pf (Cat no. 05FK50C) and CareStart HRP-2 (Cat No. G0140) RDT kits were used to test for the presence of parasite antigen in whole blood, according to the manufacturers' instructions.

\subsection{Microscopy}

Thin and thick blood films were prepared and stained using 10\% Giemsa stain. The slides were then examined under oil immersion lens $(\times 100)$. Slide examination was carried out by two microscopists. For positive slides, the number of parasites were counted against 200 leucocytes and quantified as parasite density/ $\mu \mathrm{l}$ of blood. Slides were considered negative when no parasite was detected only after examining 100 microscopic fields. Parasite density was determined using the formula below:

$$
\text { Parasites } / \mu \text { blood }=\frac{\text { Number of parasites counted } \times 8000 \text { white cells } / \mu l}{\text { No. of white cells count }}
$$

\subsection{Statistical Analysis}

The diagnostic performance characteristics such as sensitivity, specificity and 
positive \& negative predictive value (PPV \& NPV) for each kit were calculated against light microscopy. The true positive (TP), true negative (TN), false positive (FP) and false negative (FN) were used in determination of the characteristics of the kits. The formula used was, TP/TP + FN for sensitivity, TN/TN + FP for specificity, TP/TP + FP for positive predictive value (PPV) and TN/TN + FN for negative predictive value (NPV). The results were interpreted at $95 \%$ confidence interval (CI). Since both diagnostic test kits were tested on each pupil (paired data), McNemar' test was performed to determine if the sensitivities of the two diagnostic test kits were statistically different. Since $b+c<25$, McNemar' test with continuity correction [19] was used.

\subsection{Ethical Approval}

Ethical approval to conduct the study was obtained from Kenyatta National Hospital and University of Nairobi Ethics and Research Committee (Protocol P70/02/2013). Authority to conduct research was granted by the Director of Health Services, Baringo County. Written informed consent was obtained from the parents or guardians of the pupils and assent was obtained from the pupils themselves before enrolment into the study. Participation was voluntary and confidentiality was maintained. Positive individuals were treated for malaria using Artemether-Lumefantrine (AL) tablets by the study nurse who instructed the pupils to comply with treatment guidelines.

\section{Results}

During the first survey (June 2015), CareStart HRP-2, and SD Bioline Ag-Pf were evaluated. A total of 261 primary school pupils were tested out of whom 35 (13.4\%) tested positive for P. falciparum infections by both RDT kits while microscopic analysis showed that $7(2.7 \%)$ of the blood slides were positive for $P$. falciparum. The test results of both CareStart HRP-2 and SD Bioline Ag-Pf RDT kits were similar (Table 1).

\subsection{Test Performance of SD Bioline Ag-Pf and CareStart HRP-2}

The sensitivity and specificity of SD Bioline Ag-Pf and CareStart HRP-2 kits were $85.7 \%$ (95\% CL: 42.1 - 99.6) and 92.5\% (95\% CL: 88.6 - 95.4), respectively. The positive and negative predictive values (PPV and NPV) of the two kits for detection of $P$. falciparum were $24 \%$ (95\% CL: 9.36 - 4.51) and $99.6 \%$ (95\% CL: 97.7 - 100), respectively (Table 2).

Table 1. Comparative performance of RDT (CareStart HRP-2 and SD Bioline Ag-Pf) against light microscopy.

\begin{tabular}{cccc}
\hline \multirow{2}{*}{ RDT } & \multicolumn{2}{c}{ Microscopy } & \multirow{2}{*}{ Total } \\
\cline { 2 - 3 } & Positive & Negative & 35 \\
Positive & $\mathrm{TP}=6$ & $\mathrm{FP}=29$ & $\mathbf{3 5}$ \\
Negative & $\mathrm{FN}=1$ & $\mathrm{TN}=225$ & 261 \\
Total & 7 & 254 & 261 \\
\hline
\end{tabular}

Key: $\mathrm{TP}=$ True Positive, $\mathrm{FP}=$ False Positive, $\mathrm{FN}=$ False Negative, $\mathrm{TN}=$ True Negative. 
Table 2. Performance indicators of SD bioline Ag-Pf and CareStart HRP-2 kits (First survey).

\begin{tabular}{cccc}
\hline \multirow{2}{*}{ Attribute } & Test results & \multicolumn{2}{c}{$95 \%$ Confidence interval } \\
\cline { 3 - 4 } & & Lower limit & Upper limit \\
\hline Prevalence (Microscopy) & $2.7 \%$ & $1.1 \%$ & $5.45 \%$ \\
Sensitivity & $85.7 \%$ & $42.1 \%$ & $99.6 \%$ \\
Specificity & $88.6 \%$ & $84 \%$ & $92.2 \%$ \\
Likelihood ratio (+) & 7.51 & 4.75 & 11.9 \\
Likelihood ratio (-) & 0.161 & 0.0263 & 0.99 \\
Odds ratio & 46.6 & 7 & - \\
Positive predictive value & $17.1 \%$ & $6.56 \%$ & $33.6 \%$ \\
Negative predictive value & $99.6 \%$ & $97.6 \%$ & $100 \%$ \\
\hline
\end{tabular}

\subsection{Comparison between CareStart HRP-2 and SD Bioline Ag Pf/Pan}

During the second survey (October/November 2015), a total of 300 blood samples from primary school pupils were tested for the presence of Plasmodium species by CareStart HRP-2 and SD Bioline Ag-Pf/Pan. Up to 60 (20\%) pupils were diagnosed as positive for malaria. Out of the 300 blood samples, SD Bioline Ag-Pf/Pan detected 60 (20\%) samples as positive of which 45 (15\%) were positive on the $P$. falciparum band, 13 (4.3\%) positive on both $P$. falciparum and Pan band while 2 (0.7\%) were positive only on Pan band. CareStart HRP-2 diagnosed $50(16.7 \%)$ as positive with 2 invalid test results. In total, 68 (22.7\%) of 300 samples were detected as positive for Plasmodium species infections. It was however not possible to tell the two positive cases detected on pan band since the slides prepared from those two samples were read as negative by microscopy (Table 3).

\subsection{Performance of SD Bioline Ag-Pf/Pan and CareStart HRP-2}

Sensitivity, specificity and predictive values of the SD Bioline Ag-Pf/Pan and CareStart HRP-2 for detection of malaria are shown in Tables 3-6. The overall sensitivity for detection of $P$. falciparum antigen by the SD Bioline Ag-Pf/Pan was 90\% (95\% CI: 55.5 - 99.7) while that of CareStart HRP-2was 70\% (95\% CI: 34.8 - 93.3). The specificity for the SD Bioline Ag-Pf/Pan and CareStart HRP-2 was $82.4 \%$ (95\% CI: 77.5 - 86.6) and $85.2 \%$ (95\% CI: 80.6 - 89.1) respectively (Table 4 and Table 5). The study results indicate that the sensitivity of SD Bioline Ag-Pf/Pan kit was slightly higher than that of CareStart HRP-2, while the specificity of CareStart HRP-2 kit was higher than that of SD Bioline Ag-Pf/Pan, though the difference was not significant.

The positive predictive value for detection of $P$. falciparum antigen by SD Bioline Ag-Pf/Pan and CareStart HRP-2 was 15\% (95\% CI: 7.1 - 26.6) and 14\% (95\% CI: 5.82 - 26.7) respectively while the negative predictive value (NPV) for both SD Bioline Ag-Pf/Pan and CareStart HRP-2 kits was 99.6\% (95\% CI: 97.7 100) and 98.8\% (95\% CI: 96.5 - 99.8), respectively. The SD Bioline Ag-Pf/Pan 
Table 3. Malaria diagnosis by SD Bioline Ag-Pf/ Pan and Care Start HRP-2.

\begin{tabular}{lccccc}
\hline \multicolumn{5}{c}{ SD Bioline Malaria Ag P. f/ Pan (05FK6) } \\
\hline Negative & \multicolumn{5}{c}{ Positive } \\
\hline $\begin{array}{c}\text { Control } \\
\text { band only }\end{array}$ & $\begin{array}{c}P f \\
\text { band only }\end{array}$ & $\begin{array}{c}\text { Pan band } \\
\text { only }\end{array}$ & $\begin{array}{c}\text { Pf and } \\
\text { Pan band }\end{array}$ & $\begin{array}{c}\text { Total } \\
\text { positive }\end{array}$ \\
Number (\%) & $240(80)$ & $45(15)$ & $2(0.7)$ & $13(4.3)$ & $60(20)$ \\
& $\begin{array}{c}\text { Control } \\
\text { band only }\end{array}$ & $\begin{array}{c}\text { Control and } \\
P f \text { band }\end{array}$ & $\begin{array}{c}\text { Invalid } \\
\text { result }\end{array}$ & Total positive \\
Number (\%) & $248(82.7)$ & $50(16.7)$ & $2(0.7)$ & $50(16.7)$ & \\
\hline
\end{tabular}

Table 4. Comparative performance of SD Bioline Ag-Pf/Pan and Care Start HRP-2 against microscopy.

\begin{tabular}{ccccc}
\hline & & \multicolumn{3}{c}{ Microscopy } \\
\cline { 3 - 5 } & & Positive & Negative & Total \\
\hline \multirow{2}{*}{ SD Bioline Ag-Pf/Pan } & Positive & $\mathrm{TP}=9$ & $\mathrm{FP}=51$ & 60 \\
& Negative & $\mathrm{FN}=1$ & $\mathrm{TN}=239$ & 240 \\
& Total & 10 & 290 & 300 \\
CareStart HRP-2 & Positive & $\mathrm{TP}=7$ & $\mathrm{FP}=43$ & 50 \\
& Negative & $\mathrm{FN}=3$ & $\mathrm{TN}=247$ & 250 \\
Total & & 10 & 290 & 300
\end{tabular}

Key: $\mathrm{TP}=$ True Positive, $\mathrm{FP}=$ False Positive, $\mathrm{FN}=$ False Negative, $\mathrm{TN}=$ True Negative.

Table 5. Comparative performance indicators of Care Start HRP-2 and SD Bioline Ag-Pf/ $\operatorname{Pan}(\mathrm{n}=300)$.

\begin{tabular}{|c|c|c|c|}
\hline & CareStart Pf & SD Bioline Pf/ Pan & McNemar $p$ value \\
\hline $\begin{array}{c}\text { Sensitivity } \\
(95 \% \mathrm{CI})\end{array}$ & $\begin{array}{c}70 \% \\
34.8-93.3\end{array}$ & $\begin{array}{c}90 \% \\
55.5-99.7\end{array}$ & 0.4795 \\
\hline $\begin{array}{l}\text { Specificity } \\
(95 \% \mathrm{CI})\end{array}$ & $\begin{array}{c}85.2 \% \\
80.6-89.1\end{array}$ & $\begin{array}{c}\mathbf{8 2 . 4 \%} \\
77.5-86.6\end{array}$ & \\
\hline $\begin{array}{l}\text { Positive Predictive Value } \\
\qquad(95 \% \mathrm{CI})\end{array}$ & $\begin{array}{c}14 \% \\
5.82-26.7\end{array}$ & $\begin{array}{c}15 \% \\
7.1-26.6\end{array}$ & \\
\hline $\begin{array}{l}\text { Negative Predictive Value } \\
\qquad(95 \% \mathrm{CI})\end{array}$ & $\begin{array}{c}98.8 \% \\
96.5-99.8\end{array}$ & $\begin{array}{c}99.6 \% \\
97.7-100\end{array}$ & \\
\hline $\begin{array}{l}\text { Likelihood ratio (+) } \\
\text { (95\% CI) }\end{array}$ & $\begin{array}{c}4.72 \\
2.89-7.71\end{array}$ & $\begin{array}{c}5.12 \\
3.7-7.07\end{array}$ & \\
\hline $\begin{array}{l}\text { Likelihood ratio }(-) \\
\qquad(95 \% \mathrm{CI})\end{array}$ & $\begin{array}{c}0.352 \\
0.136-0.909\end{array}$ & $\begin{array}{c}0.121 \\
0.0189-0.78\end{array}$ & \\
\hline
\end{tabular}


Table 6. McNemar's statistical comparison sensitivity of SD Bioline Ag-Pf/Pan and Care Start HRP-2 kits.

\begin{tabular}{ccccc}
\hline & & \multicolumn{2}{c}{ CareStart HRP-2 } & \multirow{2}{*}{ Total } \\
\cline { 3 - 4 } & & Positive & Negative & \\
\hline \multirow{2}{*}{ SD Bioline Ag-Pf/Pan } & Positive & $\mathrm{a}=7$ & $\mathrm{~b}=2$ & 9 \\
& Negative & $\mathrm{c}=0$ & $\mathrm{~d}=1$ & 1 \\
Total & & 7 & 3 & 10 \\
\hline
\end{tabular}

McNemar's $\chi^{2}=0.5, \mathrm{df}=1, p$-value $=0.4795$.

had a positive LR of 5.12 (95\% CL: 3.7 - 7.07) and a negative LR of 0.121 (95\% CL: 0.0189 - 0.78) while that of CareStart HRP-2 was 4.72 (95\% CL: 2.89 - 7.71) and a negative LR of 0.352 (95\% CL: $0.136-0.909)$.

\subsection{McNemar's Test of Sensitivity}

The sensitivity of SD Bioline Ag-Pf/Pan (90\%) and that of CareStart HRP-2 (70\%) was tested to find out if they were significantly different. Microscopy confirmed 10 out of 300 samples as positive. Out of the 10 confirmed cases, SD Bioline Ag-Pf/Pan detected 9 as positive and 1 as negative while CareStart HRP-2 detected 7 as positive and 3 as negative. The seven samples detected by CareStart HRP-2 as positive were equally detected by SD Bioline Ag-Pf/Pan as shown in Table 6. A chi square test however showed that the sensitivities of both diagnostic kits was not significantly different $\left(\chi^{2}=0.5, \mathrm{df}=1, p=0.4795\right)$.

\subsection{Parasite Density}

Parasite density ranged between 160 to 32,800 parasites/ $\mu$ l of blood. The first survey was occasioned by relatively low parasite density of 160 - 3040 compared to second survey $(280-32,800) / \mu$ l of blood. Of the 18 positive blood slides, 8 had parasite density of less than $500 / \mu \mathrm{l}, 5$ had density below $6050 / \mu \mathrm{l}$ while the remaining 5 blood slides had parasite density ranging between $12,520 / \mu$ to 32,800 / $\mu \mathrm{l}$ of blood.

\section{Discussion}

Parasite-based diagnosis of malaria is important for prompt management. Giemsa microscopy (which detects asexual parasite stages in stained blood smear) and the serological rapid diagnostic tests (RDTs) for detection of parasite antigen represent the two diagnostics most likely to have a significant influence on malaria control [11]. During first survey a total of 261 blood samples were used to determine the performance of CareStart HRP-2 and SD Bioline Ag-Pf kits. Both kits performed well where CareStart HRP-2 and SD Bioline Pf had a sensitivity of $85.7 \%$ and specificity of $88.6 \%$. The sensitivity of CareStart HRP-2 was comparable to that reported in other studies such as $89.68 \%$ in ChinaMyanmar [20], 88.8\% in Belgium [21] and 89.1\% in Myanmar [22]. The present sensitivity was however slightly higher than that reported from an endemic re- 
gion in Nigeria at 78.4\% [23]. Moreover, the sensitivity of $85.7 \%$ for SD Bioline Ag-Pf reported in the present study was similar to the findings from a study conducted in Central African Republic where the SD Bioline Ag-Pf reported a sensitivity of $85.4 \%$ [24].

The high specificity (88.6\%) obtained in the present study for diagnosing $P$. falciparum is indicative of a specific test kit, is important for accurately detecting malaria parasite antigens with low parasite densities which might be easily missed out through microscopy [25]. This is particularly important in remote settings where adequate expert performance of microscopy may be a challenge. The high specificity findings in the present study were comparable to specificity findings reported in endemic areas of Nigeria (97.6\%) [23], 96.21\% in Karachi [26], 96\% in Sierra Leone [27] and 94.2\% in north-west Ethiopia [28], but relatively higher than $72 \%$ reported in Uganda [29]. The likelihood ratio of a test kit combines both the sensitivity and specificity into a single figure and usually indicates how the test result can reduce the uncertainty of a given diagnosis. For instance, a positive likelihood ratio of $>5$ and a negative likelihood ratio of $<0.2$ indicate high chances of a test result of a kit reporting a positive or negative outcome when given a positive screening or negative screening, respectively [30] [31]. Both CareStart and SD Bioline Pf kits had a positive LR of 7.51 and a negative LR of 0.161 . This indicated a better performance in malaria diagnosis.

There was relatively low $P$. falciparum detection by microscopy (13.3\%) in the present study compared to those reported in previous studies in Northwest Ethiopia (50.5\%) and Wondo Genet in southern Ethiopia (47\%) [32] [33]. The current findings further contradict previous studies conducted in China-Myanmar border [20] and North-West Ethiopia [28] which reported more malaria cases detected by microscopy as compared to RDTs. This disparity could however has been occasioned by the difference in the test kits used. Moreover, the low detection of $P$. falciparum by microscopy in this study could also have been due to the low parasite density as observed during the first survey of the study. The effect of low parasite density in detection of malaria parasites by microscopy was also reported by McManus and Bowles [34] and Snounou, Viriyakosol [35]. Another explanation of low detection of malaria parasites in this study could be due to auto fixation of thick smears due to long storage time before staining occasioned by the expansive nature of the study area, poor road networks and bad terrain, all of which contributed to a time lag between smear preparation in the field, and staining at the laboratory. This could have hindered the identification of parasites in thick smears which usually provide enhanced detection of parasites in blood film for easy quantification, especially in cases of low level of parasitemia [16].

During the second survey, the performance of SD Bioline Ag-Pf/Pan and CareStart HRP-2 kits were evaluated using 300 blood samples. During this period, both the sensitivity and specificity of CareStart HRP-2 slightly dropped (to 70\% and $85.2 \%$, respectively). This could have been due to the few invalid results by CareStart HRP-2, which were however positive by SD Bioline Ag-Pf and SD Bi- 
oline Ag-Pf/Pan kits. SD Bioline Ag-Pf/Pan showed a high sensitivity and specificity, similar to other studies which evaluated the performance of the same kit, like a sensitivity of $88.2 \%$ reported in the Central African Republic [24] and 97.4\% in Greece [36]. The high negative predictive values (about 99\%) for SD Bioline Ag-Pf/Pan and CareStart HRP-2 indicate their reliability in ruling out Plasmodium species infection. On the other hand, the kits showed low positive predictive values of about $15 \%$ and $14 \%$, meaning that some individuals may be falsely diagnosed as positive, hence confirmation of the positive cases by microscopy before treatment may be necessary, especially where clinical symptoms do not favour malaria diagnosis.

Two samples with high parasite densities were only detected as positive by SD Bioline Ag-Pf/Pan. Failure to detect these two samples could have been due to prozone effect which could have lowered the sensitivity of CareStart HRP-2 kits, by mechanisms earlier proposed Gillet, Mori [37]. Specifically, previous studies conducted to determine prozone effect on HRP-2-and pLDH-based kits reported prozone effects only on HRP-2-based RDT and not on PLDH based RDT [37]. Other studies also reported that sensitivity of HRP-2 based RDT kits were affected by high parasite densities ranging between 10,000 parasite/ $\mu$ l of blood to 100,000 parasite/ $\mu$ l of blood [29] [38] [39] [40] [41] [42]. The present study further reported $0.7 \%$ cases of non- $P$. falciparum plasmodium infections within the riverine zone which were not confirmed through microscopy as they all read negative. Most of the slides which were positive on both $P$. falciparum and Pan bands on the SD Bioline kits were also positive by microscopy, indicating active infection unlike HRP-2 which persists in blood long after clearance of parasites [5].

\section{Conclusion}

The findings of this study show that the performance of the three RDT kits in the diagnosis of $P$. falciparum infections is relatively adequate and can be used in guiding treatment of febrile illness in remote settings, especially where microscopy may be a challenge. Although SD Bioline Ag-Pf/Pan performed slightly better than CareStart HRP-2, the difference was not significant. Both SD Bioline Ag-Pf and CareStart HRP-2 had similar test results. The three RDT kits can reliably rule out Plasmodium species infection, although they cause false positive diagnosis of $P$. falciparum.

\section{Acknowledgements}

We thank all the children who participated in this study, parents/guardians who gave permission and support for the children's participation, and teachers for the support during enrollment, screening and treatment. We appreciate the permission from the Ministry of Education, the support of the heads and management of the schools. Appreciation also goes to the Ministry of Health Baringo County for the support, DVBD Marigat laboratory staff and the community members for their support and cooperation throughout the study period. We 
acknowledge the unequaled dedication of the field team, notably Juliet Jepkosgei, Macrae Mbalanya and Edwin Kibet.

\section{Financial Support}

This study received financial assistance from the WHO Special Programme for Research and Training in Tropical Diseases (TDR) through a grant agreement with the International Development Research Centre of Canada (106905-00).

\section{Conflict of Interests}

The authors declare that they have no conflict of interests.

\section{References}

[1] KMOH (2015) Kenya-Malaria-Operational-Plan. Kenya Ministry of Health, Kenya.

[2] MOPHS (2009) National Malaria Strategy 2009-2017. Ministry of Public Health and Sanitation, Nairobi.

[3] Mohajan, H.K. (2014) Improvement of Health Sector in Kenya. American Journal of Public Health Research, 2, 159-169. https://doi.org/10.12691/ajphr-2-4-6

[4] WHO (2008) World Malaria Report. World Health Organization, Geneva.

[5] Endeshaw, T., et al. (2008) Evaluation of Light Microscopy and Rapid Diagnostic Test for the Detection of Malaria under Operational Field Conditions: A Household Survey in Ethiopia. Malaria Journal, 7, 118. https://doi.org/10.1186/1475-2875-7-118

[6] McMorrow, M.L., et al. (2008) Challenges in Routine Implementation and Quality Control of Rapid Diagnostic Tests for Malaria-Rufiji District, Tanzania. The American Journal of Tropical Medicine and Hygiene, 79, 385-390.

[7] Mwangi, T.W., Mohammed, M., Dayo, H., Snow, R.W. and Marsh, K. (2005) Clinical Algorithms for Malaria Diagnosis Lack Utility among People of Different Age Groups. Tropical Medicine \& International Health, 10, 530-536. https://doi.org/10.1111/j.1365-3156.2005.01439.x

[8] Reyburn, H., et al. (2004) Over Diagnosis of Malaria in Patients with Severe Febrile Illness in Tanzania: A Prospective Study. BMJ, 329, 1212. https://doi.org/10.1136/bmj.38251.658229.55

[9] Snow, R.W., et al. (2009) The Coverage and Impact of Malaria Intervention in Kenya 2007-2009. Division of Malaria Control, Ministry of Health and Sanitation, Nairobi.

[10] WHO (2015) Guidelines for the Treatment of Malaria. 3rd Edition, World Health Organization, Geneva.

[11] Wongsrichanalai, C., et al. (2007) A Review of Malaria Diagnostic Tools: Microscopy and Rapid Diagnostic Test (RDT). The American Journal of Tropical Medicine and Hygiene, 77, 119-127.

[12] Amexo, M., Tolhurst, R., Barnish, G. and Bates, I. (2004) Malaria Misdiagnosis: Effects on the Poor and Vulnerable. The Lancet, 364, 1896-1898.

https://doi.org/10.1016/S0140-6736(04)17446-1

[13] Fancony, C., Sebastião, Y.V., Pires, J.E., Gamboa, D. and Nery, S.V. (2013) Performance of Microscopy and RDTs in the Context of a Malaria Prevalence Survey in Angola: A Comparison Using PCR as the Gold Standard. Malaria Journal, 12, 284. https://doi.org/10.1186/1475-2875-12-284 
[14] Biswas, S., Tomar, D. and Rao, D.N. (2005) Investigation of the Kinetics of Histidine-Rich Protein 2 and of the Antibody Responses to This Antigen, in a Group of Malaria Patients from India. Annals of Tropical Medicine and Parasitology, 99, 553562. https://doi.org/10.1179/136485905X51463

[15] Iqbal, J., Sher, A. and Rab, A. (2000) Plasmodium Falciparum Histidine-Rich Protein 2-Based Immunocapture Diagnostic Assay for Malaria: Cross-Reactivity with Rheumatoid Factors. Journal of Clinical Microbiology, 38, 1184-1186.

[16] Moody, A. (2002) Rapid Diagnostic Tests for Malaria Parasites. Clinical Microbiology Reviews, 15, 66-78. https://doi.org/10.1128/CMR.15.1.66-78.2002

[17] Hopkins, H., et al. (2007) Comparison of HRP2- and pLDH-Based Rapid Diagnostic Tests for Malaria with Longitudinal Follow-Up in Kampala, Uganda. The American Journal of Tropical Medicine and Hygiene, 76, 1092-1097.

[18] Naing, L., Winn, T. and Rusli, B.N. (2006) Practical Issues in Calculating the Sample Size for Prevalence Studies. Archives of Orofacial Sciences, 1, 6.

[19] Edwards, A.L. (1948) Note on the Correction for Continuity in Testing the Significance of the Difference between Correlated Proportions. Psychometrika, 13, 185 187. https://doi.org/10.1007/BF02289261

[20] Xiaodong, S., et al. (2013) Diagnostic Performance of CareStart Malaria HRP2/ pLDH (Pf/pan) Combo Test versus Standard Microscopy on Falciparum and Vivax Malaria between China-Myanmar Endemic Borders. Malaria Journal, 12, 6. https://doi.org/10.1186/1475-2875-12-6

[21] Maltha, J., Gillet, P., Bottieau, E., Cnops, L., van Esbroeck, M. and Jacobs, J. (2010) Evaluation of a Rapid Diagnostic Test (CareStart Malaria HRP-2/pLDH (Pf/pan) Combo Test) for the Diagnosis of Malaria in a Reference Setting. Malaria Journal, 9 , 171. https://doi.org/10.1186/1475-2875-9-171

[22] Ashley, E.A., et al. (2009) Evaluation of Three Parasite Lactate DehydrogenaseBased Rapid Diagnostic Tests for the Diagnosis of Falciparum and Vivax Malaria. Malaria Journal, 8, 241. https://doi.org/10.1186/1475-2875-8-241

[23] Sheyin, Z. and Bigwan, I.E. (2013) Comparison of Carestart HRP-2 Rapid Malaria Test with Light Microscopy for Guiding Patient's Treatment of Fever in Nigerian Endemic Areas. Medical Science, 4, 4.

[24] Djalle, D., et al. (2014) Performance of Paracheck-Pf, SD Bioline Malaria Ag-Pf and SD Bioline Malaria Ag-Pf/pan for Diagnosis of Falciparum Malaria in the Central African Republic. BMC Infectious Diseases, 14, 109.

https://doi.org/10.1186/1471-2334-14-109

[25] Guthmann, J.P., Ruiz, A., Priotto, G., Kiguli, J., Bonte, L. and Legros, D. (2002) Validity, Reliability and Ease of Use in the Field of Five Rapid Tests for the Diagnosis of Plasmodium falciparum Malaria in Uganda. Transactions of the Royal Society of Tropical Medicine and Hygiene, 96, 254-257. https://doi.org/10.1016/S0035-9203(02)90091-X

[26] Harani, M.S., et al. (2006) Role of ICT Malaria Immunochromatographic Test for Rapid Diagnosis of Malaria. Journal of the Pakistan Medical Association, 56, 167171.

[27] Gerstl, S., Dunkley, S., Mukhtar, A., De Smet, M., Baker, S. and Maikere, J. (2010) Assessment of Two Malaria Rapid Diagnostic Tests in Children under Five Years of Age, with Follow-Up of False-Positive pLDH Test Results, in a Hyperendemic Falciparum Malaria Area, Sierra Leone. Malaria Journal, 9, 28.

https://doi.org/10.1186/1475-2875-9-28

[28] Moges, B., et al. (2012) Comparison of CareStart HRP2/pLDH COMBO Rapid Ma- 
laria Test with Light Microscopy in North-West Ethiopia. Malaria Journal, 11, 234. https://doi.org/10.1186/1475-2875-11-234

[29] Kyabayinze, D.J., Tibenderana, J.K., Odong, G.W., Rwakimari, J.B. and Counihan, H. (2008) Operational Accuracy and Comparative Persistent Antigenicity of HRP2 Rapid Diagnostic Tests for Plasmodium falciparum Malaria in a Hyperendemic Region of Uganda. Malaria Journal, 7, 221. https://doi.org/10.1186/1475-2875-7-221

[30] Ashton, R.A., et al. (2010) Performance of Three Multi-Species Rapid Diagnostic Tests for Diagnosis of Plasmodium falciparum and Plasmodium vivax Malaria in Oromia Regional State, Ethiopia. Malaria Journal, 9, 297. https://doi.org/10.1186/1475-2875-9-297

[31] Akobeng, A.K. (2007) Understanding Diagnostic Tests 2: Likelihood Ratios, Preand Post-Test Probabilities and Their Use in Clinical Practice. Acta Paediatrica, 96, 487-491. https://doi.org/10.1111/j.1651-2227.2006.00179.x

[32] Hailu, T. and Kebede, T. (2014) Assessing the Performance of CareStart Malaria Pf/Pv Combo Test against Thick Blood Film in the Diagnosis of Malaria in Northwest Ethiopia. The American Journal of Tropical Medicine and Hygiene, 90, 11091112. https://doi.org/10.4269/ajtmh.13-0607

[33] Sharew, B., et al. (2009) Evaluation of the Performance of CareStart Malaria Pf/Pv Combo and Paracheck Pf Tests for the Diagnosis of Malaria in Wondo Genet, Southern Ethiopia. Acta Tropica, 111, 321-324.

https://doi.org/10.1016/j.actatropica.2009.05.014

[34] McManus, D.P. and Bowles, J. (1996) Molecular Genetic Approaches to Parasite Identification: Their Value in Diagnostic Parasitology and Systematics. International Journal for Parasitology, 26, 687-704. https://doi.org/10.1016/0020-7519(96)82612-9

[35] Snounou, G., et al. (1993) Identification of the Four Human Malaria Parasite Species in Field Samples by the Polymerase Chain Reaction and Detection of a High Prevalence of Mixed Infections. Molecular and Biochemical Parasitology, 58, 283 292. https://doi.org/10.1016/0166-6851(93)90050-8

[36] Tseroni, M., et al. (2015) Field Application of SD Bioline Malaria Ag Pf/Pan Rapid Diagnostic Test for Malaria in Greece. PLoS ONE, 10, e0120367. https://doi.org/10.1371/journal.pone.0120367

[37] Gillet, P., Mori, M., Van Esbroeck, M., Van den Ende, J. and Jacobs, J. (2009) Assessment of the Prozone Effect in Malaria Rapid Diagnostic Tests. Malaria Journal, 8, 271. https://doi.org/10.1186/1475-2875-8-271

[38] Forney, J.R., et al. (2001) Malaria Rapid Diagnostic Devices: Performance Characteristics of the ParaSight F Device Determined in a Multisite Field Study. Journal of Clinical Microbiology, 39, 2884-2890. https://doi.org/10.1128/JCM.39.8.2884-2890.2001

[39] Marx, A., et al. (2005) Meta-Analysis: Accuracy of Rapid Tests for Malaria in Travelers Returning from Endemic Areas. Annals of Internal Medicine, 142, 836-846. https://doi.org/10.7326/0003-4819-142-10-200505170-00009

[40] Ohrt, C., et al. (2007) Establishing a Malaria Diagnostics Centre of Excellence in Kisumu, Kenya. Malaria Journal, 6, 79. https://doi.org/10.1186/1475-2875-6-79

[41] Pieroni, P., Mills, C.D., Ohrt, C., Harrington, M.A. and Kain, K.C. (1998) Comparison of the ParaSight-F Test and the ICT Malaria Pf Test with the Polymerase Chain Reaction for the Diagnosis of Plasmodium falciparum Malaria in Travellers. Transactions of the Royal Society of Tropical Medicine and Hygiene, 92, 166-169. https://doi.org/10.1016/S0035-9203(98)90730-1 
[42] Van der Palen, M., Gillet, P., Bottieau, E., Cnops, L., Van Esbroeck, M. and Jacob, J. (2009) Test Characteristics of Two Rapid Antigen Detection Tests (SD FK50 and SD FK60) for the Diagnosis of Malaria in Returned Travellers. Malaria Journal, 8, 90. https://doi.org/10.1186/1475-2875-8-90

Submit or recommend next manuscript to SCIRP and we will provide best service for you:

Accepting pre-submission inquiries through Email, Facebook, LinkedIn, Twitter, etc. A wide selection of journals (inclusive of 9 subjects, more than 200 journals)

Providing 24-hour high-quality service

User-friendly online submission system

Fair and swift peer-review system

Efficient typesetting and proofreading procedure

Display of the result of downloads and visits, as well as the number of cited articles Maximum dissemination of your research work

Submit your manuscript at: http://papersubmission.scirp.org/

Or contact ojcd@scirp.org 